\title{
Faisabilité et croissance avec haute reproductibilité de multicouches métalliques à magnétorésistance géante par pulvérisation diode RF
}

\author{
J. Ben Youssef, H. Le Gall, K. Bouziane, M. El Harfaoui*, O. Koshkina, J.M. Desvignes et A. Fert** \\ CNRS-LMIMS, 92195 Meudon-Bellevue, France \\ * Université Ibn Tofail, Faculté des Sciences, BP. 133, Kenitra, Maroc \\ ** UMR, CNRS-THCSF, 91404 Orsay, France
}

\begin{abstract}
Résumé : Les objectifs du présent travail sont de deux ordres : d'abord obtenir le rapport de MRG, $\Delta R / R$, le plus élevé dans des multicouches $\mathrm{Co} / \mathrm{Cu}$ élaborées à partir du procédé inhabituel de pulvérisation par diode radiofréquence (RF). En second, analyser les corrélations entre la MRG d'une part et les textures et rugosités interfaciales déduites des diffractions X aux petits et grands angles et de la microscopie a force atomique (MFA) d'autre part. A cet effet, nous avons cherché à induire différentes structures interfaciales en modifiant les paramètres de pulvérisation (pression du gaz pulvérisant $P_{A r}$ ) ce qui, par ailleurs, a permis de définir les conditions optimales de dépôt pour l'obtention d'une

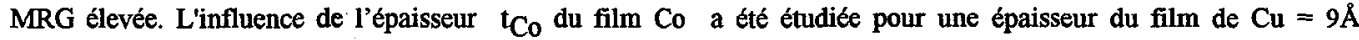
correspondant au premier maximum de la dépendance oscillatoire avec $\mathrm{t}_{\mathrm{Cu}}$ du rapport de $\mathrm{MRG}$. De ces résultats, nous concluons que le paramètre pertinent est la rugosité interfaciale qui induit un effet important à la fois sur la MRG et sur la résistivité des super-réseaux. Un changement de pente de $\mathrm{Rs}^{-1}$ en fonction de $\mathrm{t}^{2} \mathrm{Co}_{\mathrm{o}}$ est attribué à une augmentation de l'effet de rugosité quand $t_{C_{0}}$ décroît de l'échelle mésoscopique $\left(t_{C_{0}}>25 \AA\right)$ vers l'échelle nanoscopique $\left(\mathrm{t}_{\mathrm{Co}}<25 \AA\right)$.
\end{abstract}

\section{INTRODUCTION}

Malgré de nombreux travaux, tant théoriques qu'expérimentaux, publiés récemment pour comprendre l'origine physique de la magnétorésistance géante (MRG) dans les multicouches magnétiques, quelques problèmes ne sont pas complètement résolus comme l'influence de la structure interfaciale pour promouvoir la magnétorésistance (MR). Différentes hypothèses ont été présentées pour expliquer l'origine d'une MRG élevée induite par une rugosité interfaciale soit élevée ${ }^{1}$, soit faible ${ }^{2}$. Les super-réseaux $\mathrm{Co} / \mathrm{Cu}$, non seulement sont à la base de candidats excellents pour les futurs capteurs magnétiques, mais constituent aussi des structures très intéressantes pour une meilleure compréhension des propriétés de MRG. Les multicouches $\mathrm{Co} / \mathrm{Cu}$ ont été élaborées jusqu'ici soit par épitaxie par jet moléculaire, par pulvérisation par faisceau d'ions ou, pour la plupart, par pulvérisation DC magnétron. Le but de ce travail est d'une part d'obtenir le plus grand rapport $\triangle \mathrm{R} / \mathrm{R}$ de $\mathrm{MRG}$ pour les multicouches $\mathrm{Co} / \mathrm{Cu}$ élaborées par le procédé inhabituel de pulvérisation cathodique en mode diode radiofréquence (RF) et d'autre part, d'analyser en détail les corrélations entre la MRG et les textures et rugosités interfaciales, déduites de la diffraction $\mathrm{X}$ aux petits et grands angles et de la microscopie à force atomique (MFA). A cet effet, les différentes textures et rugosités d'interface ont été induites en modifiant la pression du gaz pulvérisant (pression d'argon) ce qui, par ailleurs, a permis de définir les conditions optimales de dépôt pour l'obtention d'une magnétorésistance géante.

\section{PROCEDURE EXPERIMENTALE}

Des super-réseaux $\left[\mathrm{Co}\left(\mathrm{t}_{\mathrm{Co}}\right) / \mathrm{Cu}\left(\mathrm{t}_{\mathrm{Cu}}\right)\right]_{\mathrm{n}}$ ont été déposés sur des substrats de verre après nettoyage chimique et décapage ionique, avec une couche de base (buffer) de $100 \AA$ de Fer et une couche de protection de $12 \AA$ de $C o$. Les influences de la pression d'argon, $P_{A r}$, et des épaisseurs $t_{C u}$ et $t_{C o}$ des 
couches de cuivre et de cobalt, sur la texture, la qualité du super-réseau, la résistance à saturation Rs sous champ de saturation Hs, le rapport de magnétorésistance $(\mathrm{Rm}-\mathrm{Rs}) / \mathrm{Rs}$ où $\mathrm{Rm}$ est la résistance maximum à champ nul, la sensibilité de magnétorésistance $S$ et le couplage d'échange $J$ déduit de Hs ont été analysés en détail. ${ }^{t} \mathrm{Co},{ }^{t} \mathrm{Cu}$ et $t_{\mathrm{Fe}}$ ont été déterminées à partir des vitesses de dépôt calibrées de monocouches épaisses de $\mathrm{Co}, \mathrm{Cu}$, et $\mathrm{Fe}$. De plus, le plus grand rapport de $\mathrm{MR}$ a été obtenu pour $\mathrm{t}_{\mathrm{Co}}=11 \AA$ et un nombre de périodes $\mathrm{Co} / \mathrm{Cu}$ égales à $\mathrm{n}=25$.

\section{RESULTATS ET DISCUSSION}

La figure 1 décrit la variation de la magnétorésistance des multicouches $\mathrm{Fe}(100 \AA) /\left[\mathrm{Co}(11 \AA) / \mathrm{Cu}\left(\mathrm{t}_{\mathrm{Cu}}\right)\right]_{25} / \mathrm{Co}(12 \AA)$ en fonction de l'épaisseur de la couche de $\mathrm{Cu}$ pour différentes pressions de dépôt. Pour $\mathrm{P}_{\mathrm{Ar}}>1.210^{-2} \mathrm{mbar}$, les oscillations de MRG sont bien définies avec une période de 10-12 $\AA$. Pour $\mathrm{P}_{\mathrm{Ar}}=510^{-3} \mathrm{mbar}$, la MRG augmente d'une façon monotone avec l'épaisseur de cuivre. L'absence de la première oscillation de MRG à cette pression peut être attribuée à la présence de trous ou de "ponts" couplant directement les couches de cobalt adjacentes. On note que le maximum de MRG de 43 $\%$ a été obtenu, à température ambiante, pour $\mathrm{t}_{\mathrm{Cu}}=9.5 \AA$ et $\mathrm{P}_{\mathrm{Ar}}=2.510^{-2}$ mbar. L'épaisseur de chaque couche a été contrôlée avec une très grande précision pendant les dépôts séquentiels de Co et de $\mathrm{Cu}$ comme on peut le voir sur le graphique en insertion dans la figure $1\left(\mathrm{MR}\right.$ en fonction de ${ }^{t} \mathrm{Cu}$ ), où le premier pic est obtenu pour une épaisseur extrêmement faible de cuivre de $9 \AA$.

Des cycles typiques de MR en fonction du champs, correspondant à 4 échantillons déposés à des pressions d'argon variant de $0.510^{-2}$ à $510^{-2}$ mbar, sont représentés sur la figure 2 .

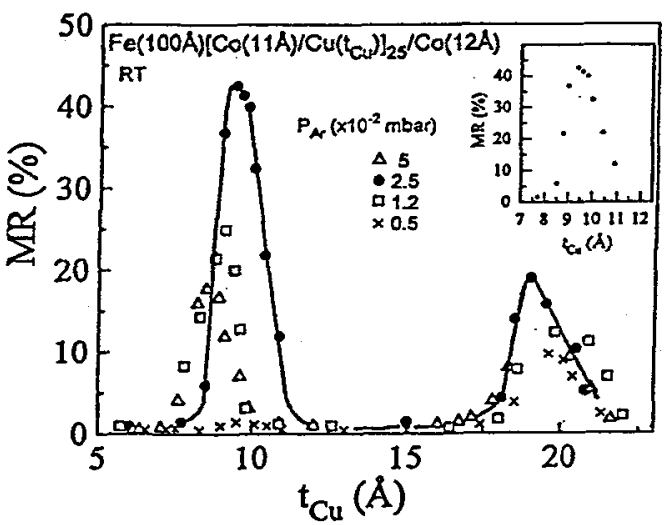

Figure 1. MR en fonction de $t_{\mathbf{C u}}$ pour les multicouches Verre/Fe(100A)/[Co(11A)/Cu(t $\mathrm{Cu})]_{25} / \mathrm{Co}(12 \mathrm{~A})$ déposées à différentes pressions d'Argon.

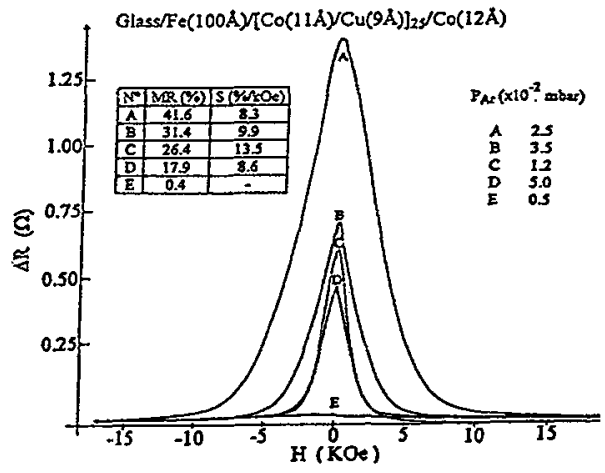

Figure 2. Cycles de MR en fonction du champ appliqué pour les multicouches $\mathrm{Fe}(100 \mathrm{~A}) /[\mathrm{Co}(11 \mathrm{~A}) / \mathrm{Cu}(9 \mathrm{~A})]_{25}$ déposées à différentes pressions d'Argon

Le facteur de rectangularité (1-Mr/Ms) des multicouches $\mathrm{Fe}(100 \AA) /\left[\mathrm{Co}(11 \AA) / \mathrm{Cu}\left(\mathrm{t}_{\mathrm{Cu}}\right)\right]_{25} / \mathrm{Co}(12 \AA)$ a été déterminé par magnétométre à échantillon vibrant à partir du cycle d'hystérésis obtenu à température ambiante (Fig.3 )

La valeur maximale du rapport de $\mathrm{MR}$, obtenue à $\mathrm{P}_{\mathrm{Ar}}=2.510^{-2} \mathrm{mbar}$, est corrélée à un minimum de résistivité, comme reporté sur la figure 4 et aussi à un maximum du champ de saturation Hs (Fig.5) proportionnel à la fois au couplage d'échange indirect entre les couches adjacentes de Co et au facteur de rectangularité (1-Mr/Ms) qui décrit qualitativement la proportion des régions couplées antiparallèlement dans le film. 


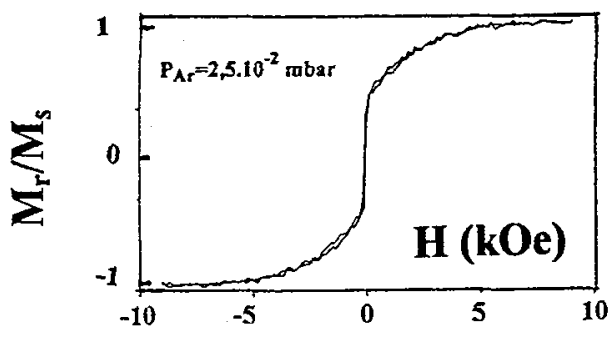

Figure 3. Cycle d'hystérésis de l'aimantation de multicouches $\mathrm{Fe}(100 \AA) /[\mathrm{Co}(11 \mathrm{~A}) / \mathrm{Cu}(9 \mathrm{~A})]_{25}$ déposés à $2.510^{2}$ mbar.

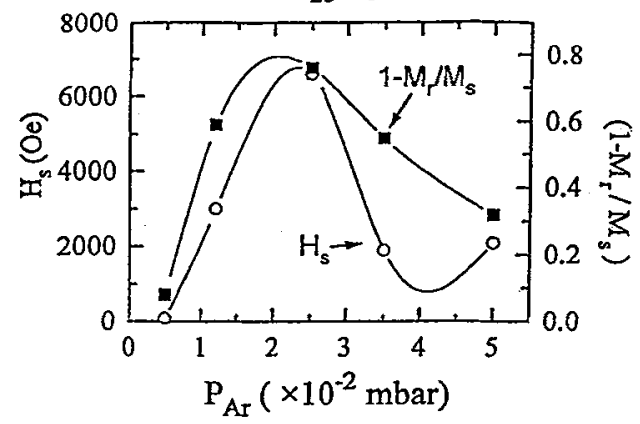

Figure 5. Hs et (1-Mr/Ms) en fonction de $\mathrm{P}_{\mathrm{ar}}$ pour les multicouches $\mathrm{Fe}(100 \AA) / \mathrm{Co}(11 \mathrm{~A}) / \mathrm{Cu}(9 \mathrm{~A})]_{25}$

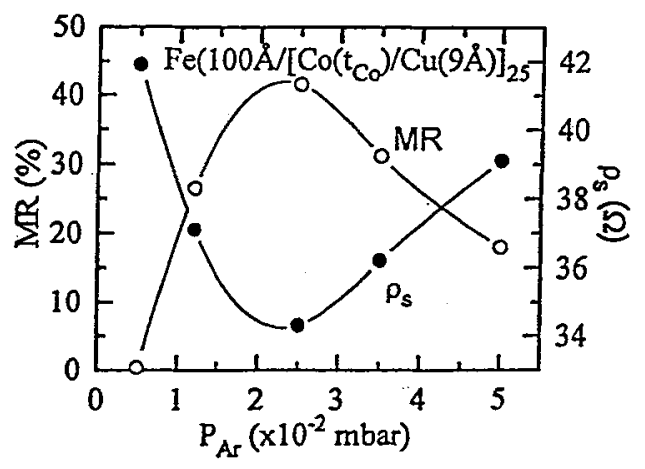

Figure 4. MR et $\rho_{S}$ en fonction de $P_{A r}$ pour les les multicouches $\mathrm{Fe}(100 \AA) /[\mathrm{Co}(11 \mathrm{~A}) / \mathrm{Cu}(9 \mathrm{~A})]_{25}$

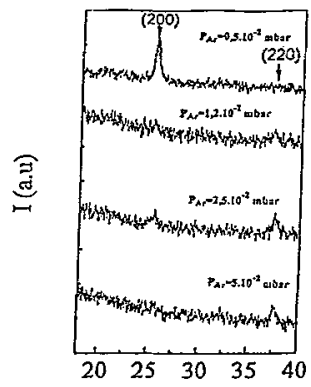

Figure 6. Spectres de diffraction $X$ aux grands angles pour pour les multicouches $\mathrm{Fe}(100 \AA) /[\mathrm{Co}(11 \mathrm{~A}) / \mathrm{Cu}(9 \mathrm{~A})]_{25}$ déposés à différentes pressions d'Argon.

La figure 6 montre les spectres de diffraction $\mathrm{X}$ aux grands angles pour les multicouches $[\mathrm{Co}(11 \mathrm{~A}) / \mathrm{Cu}(9 \mathrm{~A})]_{25}$ déposées à différentes pressions d'argon . Leur structure cristalline a été étudiée par diffractométrie $X(\theta-2 \theta)$, en utilisant une anticathode de cuivre à $1.5405 \AA$. Il est à noter que toutes nos multicouches dont l'épaisseur du cobalt varie de 8 à $62 \AA$ sont polycristallines, cristallisant dans une phase cubique à faces centrées ( Fig. 7 ). Cette conclusion diffère de celle obtenue par Shuk ${ }^{4}$ et al et Le Dang ${ }^{5}$ et al qui observent la structure hexagonale compacte (h.c.) du Co pour des épaisseurs supérieures à $40 \AA$. Cette différence est attribuée à des techniques de préparations différentes: suivant les auteurs à savoir if magnétron ${ }^{4}$, dc magnétron ${ }^{5}$ et rf diode dans notre cas. En effet la différence principale entre les deux techniques: magnétron et diode réside essentiellement dans l'échauffement du substrat. En diode le substrat est soumis à un important flux d'électrons par rapport au magnétron. L'impact de ces électrons très énergétiques sur le substrat provoque une élévation de température importante et de là une modification des propriétés cristallines des films minces. Le cobalt présente deux formes cristallines usuelles à savoir h.c stable en dessous de $400^{\circ} \mathrm{C}$ et c.f.c. au-dessus de cette température, ce qui pourrait expliquer l'absence de la structure hexagonale dans notre cas.

De plus l'orientation des multicouches dépend de la pression de pulvérisation d'argon:

A faible pression $\left(\mathrm{P}_{\mathrm{ar}}=0.510^{-2} \mathrm{mbar}\right)$, les multicouches sont bien cristallisées avec une orientation préférentielle unique (200). A forte pression ( $510^{-2} \mathrm{mbar}$ ), les multicouches sont faiblement cristallisées avec une texture (220). Aux pressions intermédiaires, où la magnétorésistance est importante, le maximum de MRG peut être corrélé à la présence simultanée des deux raies (200) et (220) d'égales intensités, contrairement aux résultats précédents qui impliquent soit une texture ${ }^{3}(200)$, soit des textures ${ }^{4}(111)$ et 
(200) dans les systèmes $\mathrm{Co} / \mathrm{Cu}$ élaborés à partir de techniques et dans des conditions de pulvérisation différentes.

Il est connu que le désordre structural dans les films brut de dépôt « as-grown » joue un rôle crucial dans une réaction de l'état solide pour former un alliage amorphe ${ }^{6}$. Les films élaborés à faible pression ne présentent pas de phase amorphe contrairement à ceux déposés à forte pression. Le désordre interfacial aurait pour effet d'activer et de promouvoir le désordre de la phase amorphe. La faible texture (220) observée dans les multicouches $\mathrm{Co} / \mathrm{Cu}$ déposée à $510^{-2}$ mbar pourrait résulter, en plus des effets déjà cités ci-dessus, d'une désorientation des grains ( grande disperssion angulaire entre les différentes cristallites) ce qui a pour conséquence d'induire une structure désordonnée avec nucléation de sites supplémentaires ${ }^{6}$.

L'analyse des spectres de diffraction $X$ en incidence rasante (Fig.8) montre la présence d'un pic de Bragg étroit et d'intensité maximale pour une pression optimale de $2.510^{-2}$ mbar correspondant au maximum de MR. Pour $P_{A r}=0.510^{-2}$ mbar, on remarque l'absence du pic de superstructure. En effet, à faibles pressions, l'énergie cinétique des atomes déposés étant très importante. Ceux-ci peuvent diffuser facilement dans la couche en cours de dépôt et former un mélange $\mathrm{Co}-\mathrm{Cu}$ à l'interface ce qui peut induire une dégradation de la superstructure.

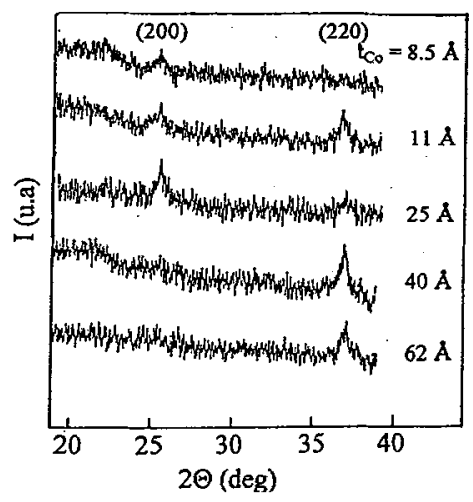

Figure 7. Spectres de diffraction $X$ aux grands angles des les multicouches $\mathrm{Fe}(100 \AA) /\left[\mathrm{Co}(11 \mathrm{~A}) / \mathrm{Cu}\left(\mathrm{t}_{\mathrm{Cu}}\right)\right]_{25}$ pour différentes épaisseurs de Co.

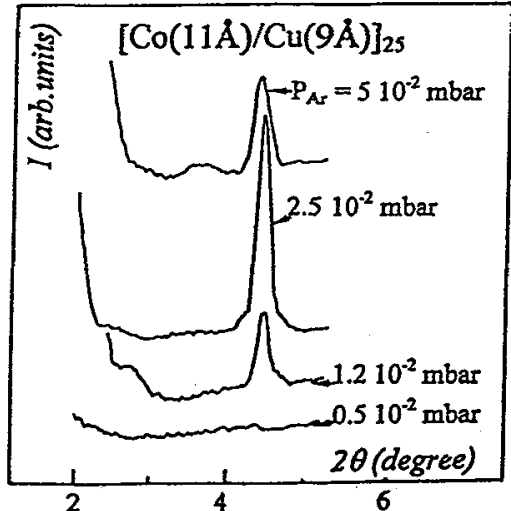

Figure 8. Spectre de diffraction $X$ en incidence rasante des multicouches $\mathrm{Fe}(100 \AA)\left[\mathrm{Co}(11 \mathrm{~A}) / \mathrm{Cu}\left(\mathrm{t}_{\mathrm{Cu}}\right)\right]_{25}$ élaborés à différentes pressions d'Argon

Les rugosités de surface définies par la valeur quadratique moyenne $\sigma_{\mathrm{rms}}$ de rugosité peuvent être déduites à partir de la largeur du pic de Bragg et par des mesures de microscopie à force atomique (MFA). Ainsi la figure 9 montre que le champ à saturation et le couplage d'échange, l'intensité du pic de Bragg et la magnétorésistance sont maximum lorsque la rugosité d'interface est minimale $\left(\sigma_{\mathrm{rms}}=3 \AA\right)$.

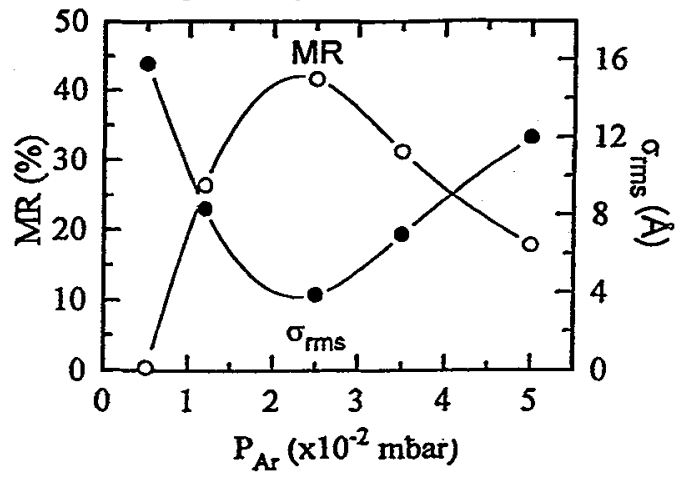

Figure 9. Variations de $M R$ et de $\sigma_{\text {rms }}$ pour les multicouches $\mathrm{Fe}(100 \AA) /[\mathrm{Co}(11 \mathrm{~A}) / \mathrm{Cu}(9 \mathrm{~A})]_{25}$ en fonction de $\mathrm{P}_{\text {ar }}$ 
Nous avons observé par ailleurs, qu'une couche de base de Fe induit le rapport de MRG le plus élevé en comparaison du Ta, Al, Cr, $\mathrm{Cu}, \mathrm{Co}, \mathrm{NiFe}$ (cristallins) ou $\mathrm{CoZr}, \mathrm{FeZr}$ (amorphes). En effet, des mesures effectuées par MFA montrent que les super-réseaux déposés sans buffer possèdent une rugosité de surface plus importante $\left(\sigma_{\mathrm{rms}}=17 \AA\right.$ et $\left.\mathrm{MR}=18 \%\right)$ que celle des films élaborés sur un buffer tel que le Fe par exemple $\left(\sigma_{\mathrm{rms}}=3 \AA ; \mathrm{MR}=43 \%\right)$, comme illustré sur les figures $10 \mathrm{a}$ et $10 \mathrm{~b}$. Ceci est associé à une bonne cristallisation de la couche de base qui induit une faible rugosité et de là , une magnétorésistance élevée.
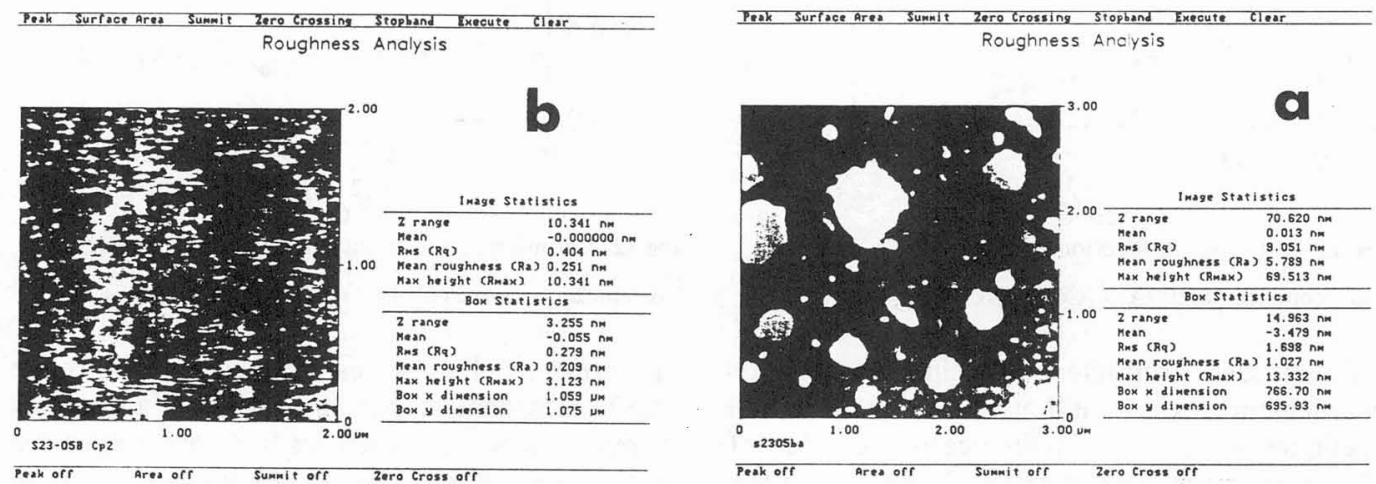

Figures 10. Rugosité observées par MFA : a) sans buffer; b) avec buffer (Fe).

La magnétorésistance est définie à partir du rapport $\Delta R / R s$ qui dépend de la variation de la résistance $\Delta R=R m-R s$ due à la différence de diffusion de spin entre des alignements antiparallèle et parallèle des aimantations des couches de Co adjacentes et de la résistance Rs de la multicouche pour un champ appliqué égal ou supérieur au champ de saturation $H s$. L'influence sur $M R$, de la diffusion dans la couche magnétique (massive) peut être analysée à partir de la dépendance de Rs en fonction de l'épaisseur de la couche de cobalt. La figure 11 montre une décroissance de Rs lorsque $t_{C o}$ augmente, ce qui n'est pas le cas de la magnétorésistance qui présente un maximum pour $\mathrm{t}_{\mathrm{Co}}=11 \AA$. A partir d'un modèle de résistances parallèles incluant les résistances, $\mathrm{R}_{\mathrm{Cu}}, \mathrm{R}_{\mathrm{Fe}}, \mathrm{R}_{\mathrm{Co}}$, des couches de $\mathrm{Cu}$, du buffer de $\mathrm{Fe}$ et de protection $\mathrm{Co}$, on montre que la conductance totale de la multicouche est décrite par la relation : $1 / \mathrm{Rs}=\mathrm{A}+\mathrm{B} \cdot \mathrm{t}_{\mathrm{Co}^{2}}$ où A est une constante dépendant des couches de base, de protection et de cuivre et $B$ est une valeur dépendant de facteurs géométriques et électriques des couches de cobalt (longueur, libre parcours moyen des électrons et résistivité du cobalt massif). Pour les films "épais", la conductance est décrite usuellement par une fonction linéaire de l'épaisseur. Par contre, pour les films de Co de très faible épaisseur, le libre parcours moyen des électrons, $\lambda_{\mathrm{Co}}$, est du même ordre de grandeur ou supérieur à l'épaisseur de $\operatorname{Co}\left(\lambda_{\mathrm{Co}}\right.$ $>_{t}$ o ). Dans ce cas la diffusion de surface contribue à la résistivité, entrainant une dépendance quadratique plutôt que linéaire de la résistivité avec $t_{\mathrm{Co}}$. Une telle dépendance quadratique est observée sur la figure 12 pour une épaisseur de Co comprise entre 25 et $70 \AA$. Ce résultat confirme la contribution croissante à la résistivité des processus de diffusion à l'interface lorsque l'épaisseur du film magnétique diminue. Le changement de pente dans la dépendance avec $t_{C o}{ }^{2}$ de $\mathrm{Rs}^{-1}$, pour $t_{C o}<25 \AA$, est attribué à une augmentation de l'effet de la rugosité interfaciale induite par la couche de Cobalt quand $t_{C o}$ décroît de l'échelle mésoscopique vers l'échelle nanoscopique. 


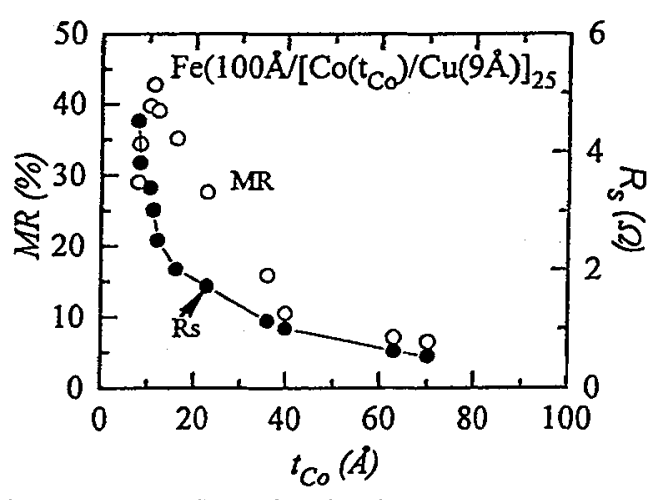

Figure 11. $M R$ et $R_{S}$ en fonction de $t_{C o}$

des multicouches $\mathrm{Fe}(100 \AA) /\left[\mathrm{Co}\left(\mathrm{t}_{\mathrm{Co}}\right) / \mathrm{Cu}(9 \mathrm{~A})\right]_{25}$.

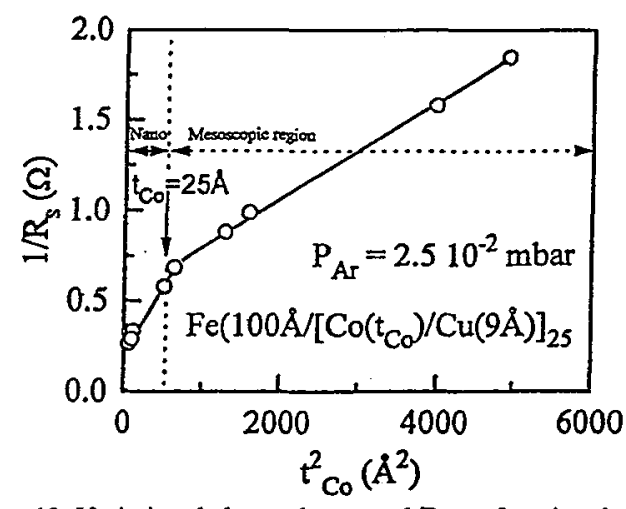

Figure 12. Variation de la conductance $1 /$ Rs en fonction de $t_{\mathrm{Co}^{2}}{ }^{2}$ des multicouches $\mathrm{Fe}(100 \AA) /\left[\mathrm{Co}\left(\mathrm{t}_{\mathrm{Co}}\right) / \mathrm{Cu}(9 \mathrm{~A})\right]_{25}$.

L'évolution des propriétés des films en fonction de la pression d'argon est associée au taux de bombardement des échantillons au cours de croissance. Le bombardement par des atomes d'argon très énergétiques favorise la mobilité des atomes à la surface, ce qui entraîne des structures très denses avec des interfaces abruptes. Les mesures de diffraction des rayons-X aux petits angles et les images de AFM indiquent cependant qu'un bombardement excessif, correspondant aux faibles pressions d'argon peut également endommager les muticouches par un accroissement de la rugosite $e^{7,8}$. Inversement une thermalisation des atomes pulvérisés aux pressions d'argon élevées entraine des structures rugueuses et moins denses de part et d'autre de la pression de dépôt optimale. Nous pouvons raisonnablement admettre l'existence d'un couplage mixte AF et $\mathrm{F}$ due aux fluctuations d'épaisseur de Co et surtout du cuivre. Cet accroissement des rugosité interfaciale, peut induire à des structures magnétiques décrites en terme de domaines antiphasiques pouvant expliquer en partie la chute de la MRG. De tels types de structure magnétiques comme observé expérimentalement par Rührig et $\mathrm{col}^{9}$ sur des sandwich épitaxiés $\mathrm{Fe}-\mathrm{Cr}$-Fe couplés AF.

\section{CONCLUSION}

Des valeurs élevées de MRG ont été obtenues dans les multicouches $\mathrm{Co} / \mathrm{Cu}$ élaborées à partir du procédé inhabituel de pulvérisation diode radiofréquence. Des corrélations entre la texture, la rugosité interfaciale dépendant de la pression du gaz pulvérisant et la MRG ont été présentées. De ces résultats, il apparaît qu'une la rugosité interfaciale minimale est requise pour promouvoir une magnétorésistance géante. Par ailleurs l'étude de la variation de MR en fonction de l'épaisseur du Co confirme la contribution croissante à la résistivité des processus de diffusion aux interfaces lorsque l'épaisseur $t_{C_{0}}$ diminue. Les études de réflectivité de neutrons à petits angles en cours pourraient nous renseigner à la fois sur le couplage indirect et le magnétisme des interfaces.

\section{Références}

[1] E.E. Fullerton, D.M. Kelly, J. Gimpel et I.K. Schuller, Phys. Rev. Letter, 68 (1992) 859.

[2] H. Veda et al., Jpn. J. Appl. Phys., 33 (1994) 6173.

[3] S. Honda, S. Ohmoto, R. Imada et M. Nawate, J. Magn. Magn. Mater., 126 (1993) 419.

[4] A.M. Shukh, D.H. Shin et H. Hoffman, J. Appl. Phys., 76 (1994) 6507.

[5] K. Le Dang, P. Veillet, E. Velu, S. S. Parkin and C. Chappert, App. Phys. Lett. 63 (1993) 108.

[6] B.M. Clemens, J. App. Phys. 61 (1987) 4525.

[7] Y. Saito, S. Hashimoto, App. Phys. Lett. 60 (1992) 2436.

[8] S. Honda, S. Ohmoto, R. Imada and M. Nawate, J. Magn. Magn. Mater. 126 (1993) 419.

[9] M. Rühring, R. Schäfer, A. Hubert, R. Mosler, J.A. Wolf, S. Demorkritov and P. Grunberg, Phys.

Status. Solidi (a) 125 (1991) 635. 